\title{
Humanoid robot NAO: review of control and motion exploration
}

\begin{abstract}
Humanoids; a most intriguing subject to behold by both the engineers and the world at large. With the introduction of humanoid robot NAO by Aldebaran-Robotics in 2008, a performant biped robot is now available and affordable for research laboratories and the mass market. In this paper, an exploration of current trends in control methods of biped walks, behavior interface tools for motion control for NAO and imminent findings in both research areas are discussed. Future directions are for researchers to devise a unique controller with low power consumption without compromising the robot's speed and robustness.
\end{abstract}

Keyword: Humanoid robot NAO; Biped walk; Control; Choregraphe 